\title{
Standard Model and New physics for $\varepsilon_{K}^{\prime} / \varepsilon_{K}$
}

\author{
Teppei Kitahara ${ }^{1,2, a}$ \\ ${ }^{1}$ Institute for Theoretical Particle Physics (TTP), Karlsruhe Institute of Technology, Engesserstraße 7, D-76128 Karlsruhe, Ger- \\ many \\ ${ }^{2}$ Institute for Nuclear Physics (IKP), Karlsruhe Institute of Technology, Hermann-von-Helmholtz-Platz 1, D-76344 Eggenstein- \\ Leopoldshafen, Germany
}

\begin{abstract}
The first result of the lattice simulation and improved perturbative calculations have pointed to a discrepancy between data on $\varepsilon_{K}^{\prime} / \varepsilon_{K}$ and the standard-model (SM) prediction. Several new physics (NP) models can explain this discrepancy, and such NP models are likely to predict deviations of $\mathcal{B}(K \rightarrow \pi v \bar{v})$ from the SM predictions, which can be probed precisely in the near future by NA62 and KOTO experiments. We present correlations between $\varepsilon_{K}^{\prime} / \varepsilon_{K}$ and $\mathcal{B}(K \rightarrow \pi v \bar{v})$ in two types of NP scenarios: a box dominated scenario and a $Z$-penguin dominated one. It is shown that different correlations are predicted and the future precision measurements of $K \rightarrow \pi v \bar{v}$ can distinguish both scenarios.
\end{abstract}

\section{Introduction and Standard-Model predictions of $\varepsilon_{K}^{\prime}$}

Charge-parity $(C P)$ violating flavour-changing neutral current decays of $K$ mesons are extremely sensitive to new physics (NP) and can probe radiative corrections of particles with masses far above the reach of the Large Hadron Collider. Prime examples of such observables are a direct $C P$ violation in $K \rightarrow \pi \pi$ decays and $\mathcal{B}\left(K_{L} \rightarrow \pi^{0} v \bar{v}\right)$.

In $K \rightarrow \pi \pi$ decays, one distinguishes between two types of $C P$ violations: direct and indirect $C P$ violations which are parametrized by $\varepsilon_{K}^{\prime}$ and $\varepsilon_{K}$, respectively. Both types of $C P$ violations have been quantified by many kaon experiments precisely. While $\varepsilon_{K}$ is a per mille effect in the Standard Model (SM), $\varepsilon_{K}^{\prime}$ is smaller by another three orders of magnitude: $\varepsilon_{K}^{\prime} \sim O\left(10^{-6}\right)$. This strong suppression comes from the smallness of the isospin-3/2 amplitude compared to the isospin- $1 / 2$ amplitude $(\Delta I=1 / 2$ rule $)$ and an accidental cancellation of leading contributions in the SM. Their suppressions lead to high sensitivity to the physics beyond the SM.

Until recently, large theoretical uncertainties precluded reliable predictions for $\varepsilon_{K}^{\prime}$. Although SM predictions of $\varepsilon_{K}^{\prime}$ using chiral perturbation theory are consistent with the experimental value, their theoretical uncertainties are large. In contrast, calculation by the dual QCD approach [1] finds the SM value much below the experimental one. A major breakthrough has been the recent lattice-QCD calculation of the hadronic matrix elements by RBC-UKQCD collaboration $[2,3]$, which gives support to the latter result.

\footnotetext{
a e-mail: teppei.kitahara@kit.edu
}

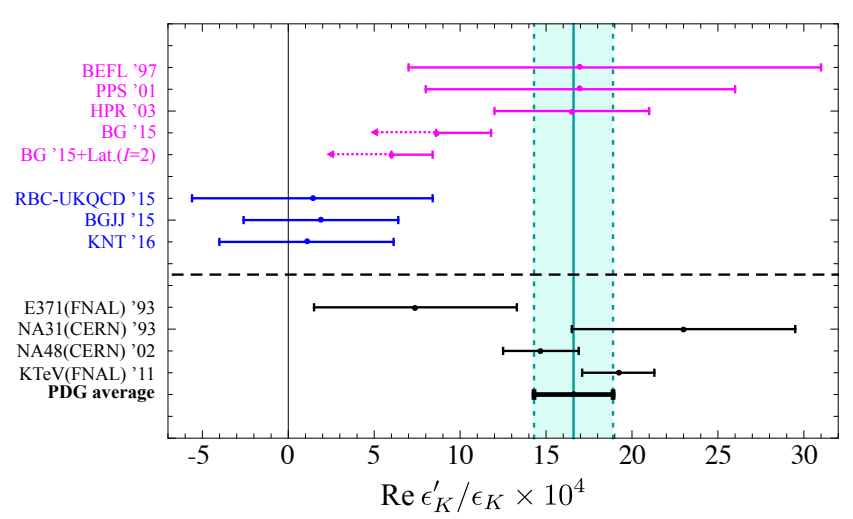

Figure 1. Compilation of representative SM predictions and the experimental values for $\operatorname{Re}\left(\varepsilon_{K}^{\prime} / \varepsilon_{K}\right)$. All error bars represent $1 \sigma$ range. The SM predictions are taken from Bertolini et al. (BEFL '97) [4], Pallante et al. (PPS '01) [5], Hambye et al. (HPR '03) [6], Buras and Gérard (BG '15) [7, 8], RBC-UKQCD lattice result [2, 3], Buras et al. (BGJJ '15) [9], and Kitahara et al. (KNT '16) [10], where magenta bars are based on analytic approaches to hadronic matrix elements, while blue bars are based on lattice results. The black thick one is the world average of the experimental values [15].

The compilation of representative SM predictions and the experimental values for $\operatorname{Re}\left(\varepsilon_{K}^{\prime} / \varepsilon_{K}\right)$ is given in Fig. 1. The SM predictions (colored bars) are taken from: Bertolini et al. (BEFL '97) [4], Pallante et al. (PPS '01) [5], Hambye et al. (HPR '03) [6], Buras and Gérard (BG'15) [7, 8] with lattice result for $I=2$ (BG'15+Lat.), RBC-UKQCD lattice result [2, 3], Buras et al. (BGJJ '15) [9], and Kitahara et al. (KNT'16) [10]. The ex- 
perimental values (black bars) are taken from: E371 [11], NA31 [12], NA48 [13] and KTeV [14] collaborations, and the black thick one is the world average of the experimental values [15]

$$
\operatorname{Re}\left(\varepsilon_{K}^{\prime} / \varepsilon_{K}\right)_{\exp }=(16.6 \pm 2.3) \times 10^{-4} .
$$

In order to predict $\varepsilon_{K}^{\prime}$ in the SM, one has to calculate the hadronic matrix elements of four-quark operators with nonperturbative methods. The magenta bars in Fig. 1 have utilized analytic approaches to calculating them: chiral quark model (BEFL '97), chiral perturbation theory (PPS '01) with minimal hadronic approximation (HPR '03), and the dual QCD approach (BG '15). Note that the dual QCD approach predicts an upper bound on $\varepsilon_{K}^{\prime} / \varepsilon_{K}$. Recently, the result of the chiral perturbation theory has been updated [16]:

$$
\left(\varepsilon_{K}^{\prime} / \varepsilon_{K}\right)_{\mathrm{ChPT}}=(15 \pm 7) \times 10^{-4}
$$

On the other hand, a determination of all hadronic matrix elements from lattice QCD has been obtained only recently by the RBC-UKQCD collaboration $[2,3]$, and the blue bars are based on the lattice result:

$$
\varepsilon_{K}^{\prime} / \varepsilon_{K}= \begin{cases}(1.9 \pm 4.5) \times 10^{-4} & \left(\mathrm{BGJJ}^{\prime} 15\right), \\ (1.06 \pm 5.07) \times 10^{-4} & \left(\mathrm{KNT}{ }^{\prime} 16\right)\end{cases}
$$

These results are obtained by next-to-leading order (NLO) calculations exploiting $C P$-conserving data to reduce hadronic uncertainties and include isospin-violating contributions [17] which are not included in the lattice result. Furthermore, the latter result includes an additional $O\left(\alpha_{E M}^{2} / \alpha_{s}^{2}\right)$ correction, which appears only in this order, and also utilizes a new analytic solution of the renormalization group (RG) equation which avoids the problem of singularities in the NLO terms. The two numbers in Eq. (3) disagree with the experimental value in Eq. (1) by $2.9 \sigma$ [9] and $2.8 \sigma$ [10], respectively. The uncertainties are dominated by the lattice statistical and systematic uncertainties for the $I=0$ amplitude.

In Fig. 2, the contributions of individual operators to $\varepsilon_{K}^{\prime} / \varepsilon_{K}$ are shown, where $y_{i}$ for $i=3,4, \ldots, 10$ are the corresponding Wilson coefficients. These values are based on Ref. [10]. $Q_{3}-Q_{6}$ are called QCD penguin operators,

$$
\begin{aligned}
Q_{3} & =(\bar{s} d)_{V-A} \sum_{q}(\bar{q} q)_{V-A}, \\
Q_{4} & =\left(\bar{s}_{\alpha} d_{\beta}\right)_{V-A} \sum_{q}\left(\bar{q}_{\beta} q_{\alpha}\right)_{V-A}, \\
Q_{5} & =(\bar{s} d)_{V-A} \sum_{q}(\bar{q} q)_{V+A}, \\
Q_{6} & =\left(\bar{s}_{\alpha} d_{\beta}\right)_{V-A} \sum_{q}\left(\bar{q}_{\beta} q_{\alpha}\right)_{V+A},
\end{aligned}
$$

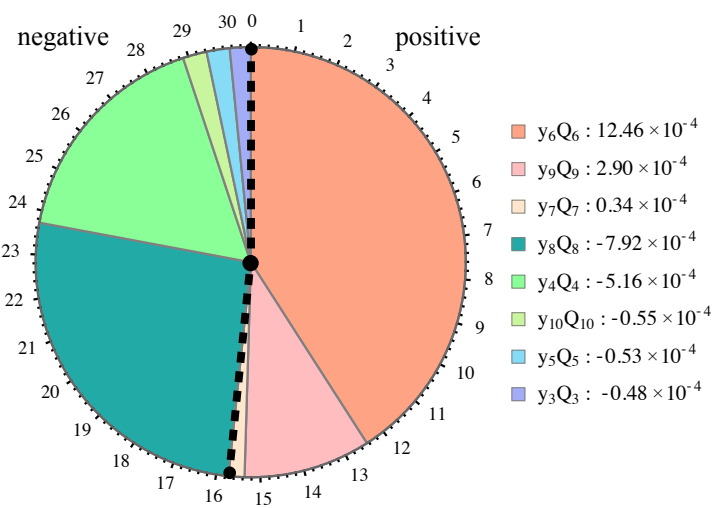

Figure 2. The composition of $\varepsilon_{K}^{\prime} / \varepsilon_{K}$ with respect to the operator basis. The right and left side of the dashed lines represent positive and negative contributions to $\varepsilon_{K}^{\prime} / \varepsilon_{K}$, respectively. This figure is based on the result of Ref. [10].

while $Q_{7}-Q_{10}$ are called electroweak penguin operators,

$$
\begin{aligned}
Q_{7} & =\frac{3}{2}(\bar{s} d)_{V-A} \sum_{q} e_{q}(\bar{q} q)_{V+A}, \\
Q_{8} & =\frac{3}{2}\left(\bar{s}_{\alpha} d_{\beta}\right)_{V-A} \sum_{q} e_{q}\left(\bar{q}_{\beta} q_{\alpha}\right)_{V+A}, \\
Q_{9} & =\frac{3}{2}(\bar{s} d)_{V-A} \sum_{q} e_{q}(\bar{q} q)_{V-A}, \\
Q_{10} & =\frac{3}{2}\left(\bar{s}_{\alpha} d_{\beta}\right)_{V-A} \sum_{q} e_{q}\left(\bar{q}_{\beta} q_{\alpha}\right)_{V-A},
\end{aligned}
$$

where $V \mp A$ represents $\gamma_{\mu}\left(1 \mp \gamma_{5}\right), \alpha$ and $\beta$ denote color indices, and $e_{q}$ is the electric charge of the quark $q$. The leading contributions come from $Q_{6}$ and $Q_{8}$, having opposite sign, and thus a cancellation emerges. Remarkably, this figure also shows that even if one includes sub-leading contributions, the cancellation still exists with high precision.

The main difference between each result of analytic approaches and the lattice result comes from the hadronic parameter $B_{6}^{(1 / 2)} \propto\left\langle(\pi \pi)_{I=0}\left|Q_{6}\right| K^{0}\right\rangle$, which controls the largest positive contribution to $\varepsilon_{K}^{\prime} / \varepsilon_{K}$ (the $y_{6} Q_{6}$ component in the Fig. 2). In chiral perturbation theory, typically large values are obtained: $B_{6}^{(1 / 2)} \sim 1.6$ (BEFL '97), $~ 1.6$ (PPS '01), and $\sim 3$ (HPR '03, see Ref. [7]). On the other hand, the dual QCD approach predicts a smaller number, $B_{6}^{(1 / 2)} \leq B_{8}^{(3 / 2)} \sim 0.8\left(\mathrm{BG}^{\prime} 15\right)$. The lattice result is consistent with the latter result: $B_{6}^{(1 / 2)}=0.56 \pm 0.20[3,10]$. Although the lattice simulation [3] includes final-state interactions partially along the line of Ref. [18], final-state interactions have to be still fully included in the calculations in light of a discrepancy of a strong phase shift $\delta_{0}[19,20]$. In the near future, the increasing precision of lattice calculations with improved methods will further sharpen the SM predictions in Eq. (3) and answer the question about NP in $\varepsilon_{K}^{\prime} / \varepsilon_{K}[21]$. 
We also should comment on the $\Delta I=1 / 2$ rule $\left(\operatorname{Re} A_{0} / \operatorname{Re} A_{2}\right)_{\text {exp. }}=22.45 \pm 0.05$. Although none of the analytic approaches can explain such a large value, the first lattice calculation has found a consistent value within $1 \sigma$, $\left(\operatorname{Re} A_{0} / \operatorname{Re} A_{2}\right)_{\text {Lat. }}=31.0 \pm 11.1[2,3,8]$.

Several NP models including supersymmetry (SUSY) can explain the discrepancy of $\varepsilon_{K}^{\prime} / \varepsilon_{K}$. It is known that such NP models are likely to predict deviations of branching ratios of the kaon rare decay from the SM predictions, especially $\mathcal{B}(K \rightarrow \pi v \bar{v})$ which includes $C P$-violating flavour-changing neutral current decay and can be probed precisely in the near future by NA62 and KOTO experiments. ${ }^{1}$ In this contribution, based on the lattice result of $\varepsilon_{K}^{\prime} / \varepsilon_{K}$ and Eq. (3), we present correlations between $\varepsilon_{K}^{\prime} / \varepsilon_{K}$ and $\mathcal{B}(K \rightarrow \pi v \bar{v})$ in two types of NP scenarios: a box dominated scenario and a $Z$-penguin dominated one, and discuss how to distinguish between them.

\section{Box dominated (Trojan penguin) scenario}

We first focus on the box dominated scenario, where all NP contributions to $|\Delta S|=1$ and $|\Delta S|=2$ processes are dominated by the four-fermion box diagrams. Such a situation is realized in the minimal supersymmetric standard model (MSSM) [23]. The desired effect in $\varepsilon_{K}^{\prime}$ is generated via gluino-squark box diagrams which are shown in Fig. 3, when the mass difference between the right-handed up and down squarks exists. Such a contribution is called Trojan penguin because its effect is parameterized by the electroweak penguin operator at low energy scale [24].

While the sizable effects in $\varepsilon_{K}^{\prime}$ are obtained by the Trojan penguin contributions, a simultaneous efficient suppression of the SUSY QCD contributions to $\varepsilon_{K}$ can also be achieved, which is shown in the following subsection.

\subsection{Suppression of contributions to $\varepsilon_{K}$}

An explanation of the puzzle between Eq. (1) and Eq. (3) by physics beyond the SM requires a NP contribution which is seemingly even larger than the SM contribution. However, it is known that once constraints from the corresponding $|\Delta S|=2$ transition are taken into account, one expects that NP effects in a $|\Delta S|=1$ four-quark process are highly suppressed. To explain the NP hierarchy in $|\Delta S|=1$ vs $|\Delta S|=2$ transitions, we specify to $\varepsilon_{K}^{\prime}$ and $\varepsilon_{K}$ : The SM contributions are governed by the combination $\tau=-V_{t d} V_{t s}^{*} /\left(V_{u d} V_{u s}^{*}\right) \sim(1.5-i 0.6) \times 10^{-3}$ with $\varepsilon_{K}^{\prime S M} \propto \operatorname{Im} \tau / M_{W}^{2}$ and $\varepsilon_{K}^{\mathrm{SM}} \propto \operatorname{Im} \tau^{2} / M_{W}^{2}$. If the NP contribution enters through the $|\Delta S=1|$ parameter $\delta$ and is mediated by heavy particles of mass $M$, one obtains $\varepsilon_{K}^{\prime \mathrm{NP}} \propto \operatorname{Im} \delta / M^{2}, \varepsilon_{K}^{\mathrm{NP}} \propto \operatorname{Im} \delta^{2} / M^{2}$, and therefore the experimental constraint $\left|\varepsilon_{K}^{\mathrm{NP}}\right| \leq\left|\varepsilon_{K}^{\mathrm{SM}}\right|$ leads to

$$
\left|\frac{\varepsilon_{K}^{\prime \mathrm{NP}}}{\varepsilon_{K}^{\prime \mathrm{SM}}}\right| \leq \frac{\left|\varepsilon_{K}^{\prime \mathrm{NP}} / \varepsilon_{K}^{\prime \mathrm{SM}}\right|}{\left|\varepsilon_{K}^{\mathrm{NP}} / \varepsilon_{K}^{\mathrm{SM}}\right|}=O\left(\frac{\operatorname{Re} \tau}{\operatorname{Re} \delta}\right)
$$

${ }^{1}$ The correlations between $\varepsilon_{K}^{\prime} / \varepsilon_{K}, \mathcal{B}(K \rightarrow \pi v \bar{v})$, and $\varepsilon_{K}$ through the CKM components in the SM are discussed in Ref. [22].

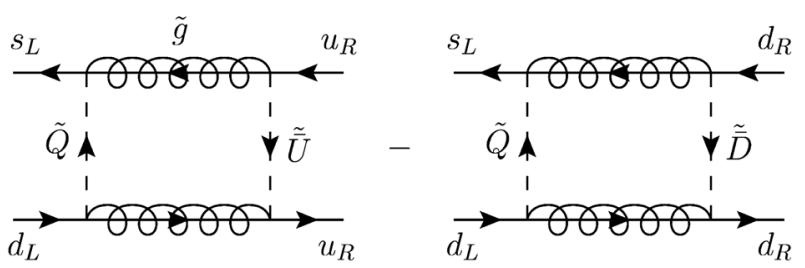

Figure 3. Trojan penguin contributions to $\operatorname{Im} A_{2}$ for $m_{\bar{U}} \neq m_{\bar{D}}$.

If NP enters through a loop with particles of mass $M \gtrsim 1$ $\mathrm{TeV}$, the NP effects can be relevant only for $|\delta| \gg|\tau|$, and thus Eq. (12) seemingly forbids detectable NP contributions to $\varepsilon_{K}^{\prime}$.

In the MSSM, there is a bypass to Eq. (12). The Majorana nature of the gluino leads to a suppression of gluino-squark box contributions to $\varepsilon_{K}$. This is so, because there are two such diagrams (crossed and uncrossed boxes) with opposite signs. If the gluino mass $m_{\tilde{g}}$ equals roughly 1.5 times the average down squark mass $M_{S}$, both contributions to $\varepsilon_{K}^{\text {SUSY }}$ cancel [25]. For $m_{\tilde{g}}>1.5 M_{S}$, the gluino-box contribution approximately behaves as $\left[m_{\tilde{g}}^{2}-\left(1.5 M_{S}\right)^{2}\right] / m_{\tilde{g}}^{4}$, and the $1 / m_{\tilde{g}}^{2}$ decoupling sets in. Note that this suppression appears only when a hierarchy $\Delta_{Q, 12} \gg \Delta_{\bar{D}, 12}$ of $\Delta_{Q, 12} \ll \Delta_{\bar{D}, 12}$ is satisfied, where the following notation is used for the squark mass matrices: $M_{X, i j}^{2}=m_{X}^{2}\left(\delta_{i j}+\Delta_{X, i j}\right)$, with $X=Q, \bar{U}$, or $\bar{D}$.

\subsection{Contributions to $\varepsilon_{K}^{\prime}$}

The master equation for $\varepsilon_{K}^{\prime} / \varepsilon_{K}$ (see e.g. Ref. [9]) reads:

$$
\frac{\varepsilon_{K}^{\prime}}{\varepsilon_{K}}=\frac{\omega_{+}}{\sqrt{2}\left|\varepsilon_{K}^{\exp }\right| \operatorname{Re} A_{0}^{\exp }}\left[\frac{\operatorname{Im} A_{2}}{\omega_{+}}-\left(1-\hat{\Omega}_{\mathrm{eff}}\right) \operatorname{Im} A_{0}\right],
$$

with $\hat{\Omega}_{\mathrm{eff}}=(14.8 \pm 8.0) \times 10^{-2}$, the measured $\left|\varepsilon_{K}^{\exp }\right|$, $\omega_{+}=(4.53 \pm 0.02) \times 10^{-2}$, and the amplitudes $A_{I}=$ $\left\langle(\pi \pi)_{I}\left|\mathcal{H}^{|\Delta S|=1}\right| K^{0}\right\rangle$ involving the effective $|\Delta S|=1$ Hamiltonian $\mathcal{H}^{|\Delta S|}$. $I=0,2$ represents the strong isospin of the final two-pion state.

The MSSM contributions to $\varepsilon_{K}^{\prime} / \varepsilon_{K}$ have been widely studied in the past. However, the SUSY-breaking scale $M_{S}$ was considered in the ballpark of the electroweak scale, so that the suppression mechanism inferred from Eq. (12) is avoided. The low-energy Hamiltonian in the case of small left-right squark mixing reads

$$
\begin{aligned}
\mathcal{H}_{\mathrm{eff}, \text { SUSY }}^{|\Delta S|=1}= & \frac{G_{F}}{\sqrt{2}} \sum_{q}\left[\sum_{i=1}^{2} c_{i}^{q}(\mu) Q_{i}^{q}(\mu)\right. \\
& +\sum_{i=1}^{4}\left[c_{i}^{\prime q}(\mu) Q_{i}^{\prime q}(\mu)+\tilde{c}_{i}^{\prime q}(\mu) \tilde{Q}_{i}^{\prime q}(\mu)\right]+\text { H.c. }
\end{aligned}
$$


where $G_{F}$ is the Fermi constant and

$$
\begin{aligned}
Q_{1}^{q=u, c, t} & =\left(\bar{s}_{\alpha} q_{\beta}\right)_{V-A}\left(\bar{q}_{\beta} d_{\alpha}\right)_{V-A}, \\
Q_{2}^{q=u, c, t} & =(\bar{s} q)_{V-A}(\bar{q} d)_{V-A}, \\
Q_{1}^{\prime q=a l l} & =(\bar{s} d)_{V-A}(\bar{q} q)_{V+A}, \\
Q_{2}^{\prime q=a l l} & =\left(\bar{s}_{\alpha} d_{\beta}\right)_{V-A}\left(\bar{q}_{\beta} q_{\alpha}\right)_{V+A}, \\
Q_{3}^{\prime q=a l l} & =(\bar{s} d)_{V-A}(\bar{q} q)_{V-A}, \\
Q_{4}^{\prime q=a l l} & =\left(\bar{s}_{\alpha} d_{\beta}\right)_{V-A}\left(\bar{q}_{\beta} q_{\alpha}\right)_{V-A} .
\end{aligned}
$$

Here, opposite-chirality operators $\tilde{Q}_{i}^{\prime q}$ are given by interchanging $V-A \leftrightarrow V+A$.

In this section, we focus on a case that the dominant SUSY contribution comes from Trojan penguin diagrams in Fig. 3. The other SUSY solution focusing the Z-penguin contributions is investigated in the next section. The box diagrams contribute to $\operatorname{Im} A_{2}$ when $m_{\bar{U}} \neq m_{\bar{D}}$. Because these contributions are governed by the strong interaction and there is an enhancement factor $1 / \omega_{+}=22.1$ for the $\operatorname{Im} A_{2}$ term in (13), they easily become the largest contribution to $\varepsilon_{K}^{\prime} / \varepsilon_{K}$ [24]. In order to obtain the desired large effect in $\varepsilon_{K}^{\prime}$, one needs a contribution to the operators $Q_{1,2}^{\prime}$ with $(V-A) \times(V+A)$ Dirac structure, whose matrix elements are chirally enhanced by a factor $\left(m_{K} / m_{s}\right)^{2}$. Hence, the flavour mixing has to be in the left-handed squark mass matrix. The opposite situation with right-handed flavour mixing and $\tilde{u}_{L}-\tilde{d}_{L}$ mass splitting is not possible because of the $\mathrm{SU}(2)_{L}$ invariance.

For the calculation of SUSY contributions to $\varepsilon_{K}^{\prime} / \varepsilon_{K}$, one has to use the RG equations to evolve the Wilson coefficients calculated at the high scale $M_{S}$ down to the hadronic scale $\mu_{h}=1.3 \mathrm{GeV}$ at which the hadronic matrix elements are calculated $[2,3,10]$. To use the wellknown NLO $10 \times 10$ anomalous dimensions for the SM four-fermion operator basis [26], we switch from Eq. (14) to

$$
\mathcal{H}_{\mathrm{eff}, \mathrm{SUSY}}^{|\Delta S|=1}=\frac{G_{F}}{\sqrt{2}} \sum_{i=1}^{10}\left[C_{i}(\mu) Q_{i}(\mu)+\tilde{C}_{i}(\mu) \tilde{Q}_{i}(\mu)\right]+\text { H.c. }
$$

where $Q_{1, \ldots, 10}$ are given in Eqs. (4)-(11), (15), and (16), and

$$
\begin{aligned}
C_{1,2}(\mu) & =c_{1,2}^{u}(\mu), \\
\tilde{C}_{1,2}(\mu) & =0, \\
C_{3,4,5,6}(\mu) & =\frac{1}{3}\left[c_{3,4,1,2}^{\prime u}(\mu)+2 c_{3,4,1,2}^{\prime d}(\mu)\right], \\
C_{7,8,9,10}(\mu) & =\frac{2}{3}\left[c_{1,2,3,4}^{\prime u}(\mu)-c_{1,2,3,4}^{\prime d}(\mu)\right],
\end{aligned}
$$

and the coefficients $\tilde{C}_{3, \ldots 10}$ for the opposite-chirality operators can be obtained from $C_{3, \ldots 10}$ by replacing $c_{i}^{q} \rightarrow \tilde{c}_{i}^{\prime}$. Note that the contribution of Fig. 3 is collected into the coefficients $C_{7,8}$. For the RG evolution of the coefficients, we use the new analytic solution of the RG equations discussed in Ref. [10].

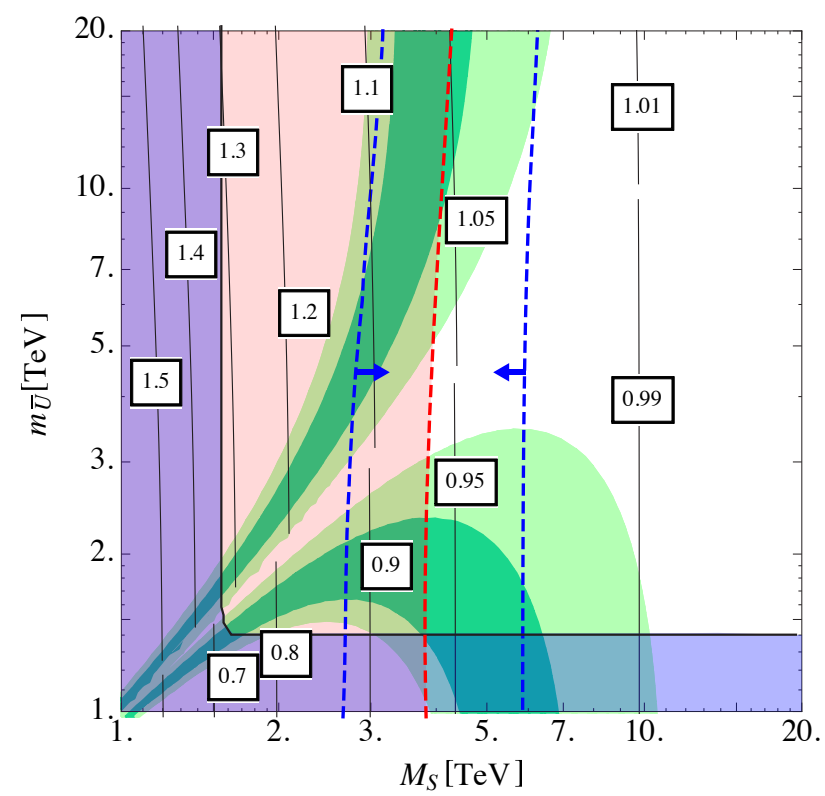

Figure 4. The $\varepsilon_{K}^{\prime} / \varepsilon_{K}$ discrepancy between Eq. (1) and Eq. (3) is resolved at the $1 \sigma(2 \sigma)$ level within the dark (light) green region. The red shaded region is excluded by $\varepsilon_{K}$ at $95 \%$ C.L. using the inclusive value $\left|V_{c b}\right|$, while the region between the blue-dashed lines can explain the $\varepsilon_{K}$ discrepancy which is present if the exclusive determination of $\left|V_{c b}\right|$ is used [27]. The blue shaded region is excluded by the current LHC results from CMS and ATLAS [2830]. The only nonzero off-diagonal element of the squark mass matrices is $\Delta_{Q, 12}=0.1 \exp (-i \pi / 4)$ for $m_{\bar{U}}>m_{\bar{D}}=M_{S}$ (upper branch) and $\Delta_{Q, 12}=0.1 \exp (i 3 \pi / 4)$ for $m_{\bar{U}}<m_{\bar{D}}=M_{S}$ (lower branch). Black contour represents $\mathcal{B}\left(K_{L} \rightarrow \pi^{0} v \bar{v}\right) / \mathcal{B}^{\mathrm{SM}}\left(K_{L} \rightarrow\right.$ $\left.\pi^{0} v \bar{v}\right)$.

In Fig. 4, the portion of the squark mass plane which simultaneously explains $\varepsilon_{K}^{\prime} / \varepsilon_{K}$ discrepancy and $\varepsilon_{K}$ constraint is shown. As input, we take the grand-unified theory $(\mathrm{GUT})$ relation for gaugino masses, $\alpha_{s}\left(M_{Z}\right)=0.1185$, $m_{\tilde{g}} / M_{S}=1.5$ for the suppressed $\varepsilon_{K}$, and $m_{Q}=m_{\bar{D}}=$ $\mu_{\text {SUSY }}=M_{S}$ with varying $m_{\bar{U}}$. The universal slepton mass is set to be $m_{L}=300 \mathrm{GeV}$. Furthermore, the trilinear SUSY-breaking matrices $A_{q}$ are set to zero, $\tan \beta=10$, and the only nonzero off-diagonal element of the squark mass matrices is $\Delta_{Q, 12}=0.1 \exp (-i \pi / 4)$ for the lefthanded squark sectors for $m_{\bar{U}}>m_{\bar{D}}=M_{S}$ (upper branch) and $\Delta_{Q, 12}=0.1 \exp (i 3 \pi / 4)$ for $m_{\bar{U}}<m_{\bar{D}}=M_{S}$ (lower branch). We have calculated all relevant one-loop contributions to the coefficients in Eq. (14) in the squark mass eigenbasis. The $\varepsilon_{K}^{\prime} / \varepsilon_{K}$ discrepancy between Eq. (1) and Eq. (3) can be resolved at $1 \sigma(2 \sigma)$ in the dark (light) green region. The red region is excluded by the measurement of $\varepsilon_{K}$ at $95 \%$ C.L. in combination with the inclusive $\left|V_{c b}\right|$, while the region between the blue dashed lines can explain the $\varepsilon_{K}$ discrepancy at $95 \%$ C.L. for the exclusive value of $\left|V_{c b}\right|$ [27]. Note that $\theta= \pm \pi / 4$ maximizes the effect in $\varepsilon_{K}^{\text {SUSY }}$, while the SUSY contributions to $\varepsilon_{K}^{\prime} / \varepsilon_{K}$ is maximized at $\theta= \pm \pi / 2$ resulting instead in a vanishing effect 
in $\varepsilon_{K}^{\text {SUSY }}$. Therefore, Fig. 4 is a conservative result in light of the $C P$-violating phase.

The blue shaded region is excluded by the current LHC results [28-30]. Here, in order to be conservative, we use the most stringent one, i.e. we maximize the bound which is a function of the neutralino mass.

The black contour represents $\mathcal{B}\left(K_{L} \rightarrow \pi^{0} v \bar{v}\right)$ which is normalized by $\mathcal{B}^{\mathrm{SM}}\left(K_{L} \rightarrow \pi^{0} v \bar{v}\right)=(2.9 \pm 0.2) \times 10^{-11}$ [31]. In this setup, we find that $\mathcal{B}\left(K_{L} \rightarrow \pi^{0} v \bar{v}\right) / \mathcal{B}^{\mathrm{SM}}\left(K_{L} \rightarrow\right.$ $\left.\pi^{0} v \bar{v}\right) \simeq 1.05-1.1$ is predicted in light of the $\varepsilon_{K}^{\prime} / \varepsilon_{K}$ discrepancy (and the potential $\varepsilon_{K}$ discrepancy) if $m_{\bar{U}}>m_{\bar{D}}$. More detailed investigation of $\mathcal{B}\left(K_{L} \rightarrow \pi^{0} v \bar{v}\right)$ is shown in the next subsection.

\section{$2.3 \mathcal{B}\left(K_{L} \rightarrow \pi^{0} v \bar{v}\right)$ and $\mathcal{B}\left(K^{+} \rightarrow \pi^{+} v \bar{v}\right)$}

The SUSY contributions to $\varepsilon_{K}$ can be suppressed by the crossed and uncrossed box diagrams when the gluino mass is heavier than the squark mass, while there is no such cancellation in a chargino box contribution to $K_{L} \rightarrow \pi^{0} v \bar{v}$ and $K^{+} \rightarrow \pi^{+} v \bar{v}$ which permits potentially large effects. We investigate the correlation between $\varepsilon_{K}^{\prime}$ and $\mathcal{B}(K \rightarrow \pi v \bar{v})$ varying the following parameters:

$$
\left|\Delta_{Q, 12}\right|, \theta, M_{3}, m_{\bar{U}} / m_{\bar{D}}
$$

with $0<\left|\Delta_{Q, 12}\right|<1$ and $0<\theta<2 \pi$. Here, defining the bilinear terms for the squarks as $\theta \equiv \arg \left(\Delta_{Q, 12}\right)$. We fix the slepton mass and the lightest squark mass close to the experimental limit $\left(m_{L}=300 \mathrm{GeV}\right.$ and $\left.m_{\tilde{q}_{1}}=1.5 \mathrm{TeV}\right)$ and use GUT relations among all three gaugino masses.

Figure 5 shows the correlations between $\varepsilon_{K}^{\prime}$ and $\mathcal{B}(K \rightarrow \pi v \bar{v})$ in the $\mathcal{B}\left(K_{L} \rightarrow \pi^{0} v \bar{v}\right)-\mathcal{B}\left(K^{+} \rightarrow \pi^{+} v \bar{v}\right)$ plane which are normalized by their SM predictions [31]. We find that the necessary amount of the tuning in the gluino mass and the $C P$ violating phase in order to suppress contributions to $\varepsilon_{K}$ determines deviations of $\mathcal{B}(K \rightarrow \pi v \bar{v})$ from the SM values. A quantity which parameterizes the fine-tuning parameter is defined in Ref. [31]. The current $\varepsilon_{K}^{\prime} / \varepsilon_{K}$ discrepancy between Eq. (1) and Eq. (3) is resolved at $1 \sigma(2 \sigma)$ within the dark (light) green region. In the top (bottom) panel we used $m_{\bar{D}} / m_{\bar{U}}=1.1$ (2) with $m_{\bar{U}}=m_{Q}$ for $0<\theta<\pi$, and $m_{\bar{U}} / m_{\bar{D}}=1.1$ (2) with $m_{\bar{D}}=m_{Q}$ for $\pi<\theta<2 \pi$. Numerically, we observe $\mathcal{B}\left(K_{L} \rightarrow \pi^{0} v \bar{v}\right) / \mathcal{B}^{\mathrm{SM}}\left(K_{L} \rightarrow \pi^{0} v \bar{v}\right) \lesssim 2(1.2)$ and $\mathcal{B}\left(K^{+} \rightarrow \pi^{+} v \bar{v}\right) / \mathcal{B}^{\mathrm{SM}}\left(K^{+} \rightarrow \pi^{+} v \bar{v}\right) \lesssim 1.4(1.1)$ in light of $\varepsilon_{K}^{\prime} / \varepsilon_{K}$ discrepancy, if all squarks are heavier than $1.5 \mathrm{TeV}$ and if a $1(10) \%$ fine-tuning is permitted.

We also observe a strict correlation between $\mathcal{B}\left(K_{L} \rightarrow\right.$ $\left.\pi^{0} v \bar{v}\right)$ and $m_{\bar{U}} / m_{\bar{D}}: \quad \operatorname{sgn}\left[\mathcal{B}\left(K_{L} \rightarrow \pi^{0} v \bar{v}\right)-\mathcal{B}^{\mathrm{SM}}\left(K_{L} \rightarrow\right.\right.$ $\left.\left.\pi^{0} v \bar{v}\right)\right]=\operatorname{sgn}\left[m_{\bar{U}}-m_{\bar{D}}\right]$. Thus, $\mathcal{B}\left(K_{L} \rightarrow \pi^{0} v \bar{v}\right)$ can indirectly determine whether the right-handed up or down squark is the heavier one.

\section{Z-penguin dominated (modified $Z$-coupling) scenario}

Next, we focus on the $Z$-penguin dominated scenario. The negative dominant contribution to $\varepsilon_{K}^{\prime} / \varepsilon_{K}$ comes from $Z$ penguin diagrams in the SM as $y_{8} Q_{8}$ (see Fig. 2). Since
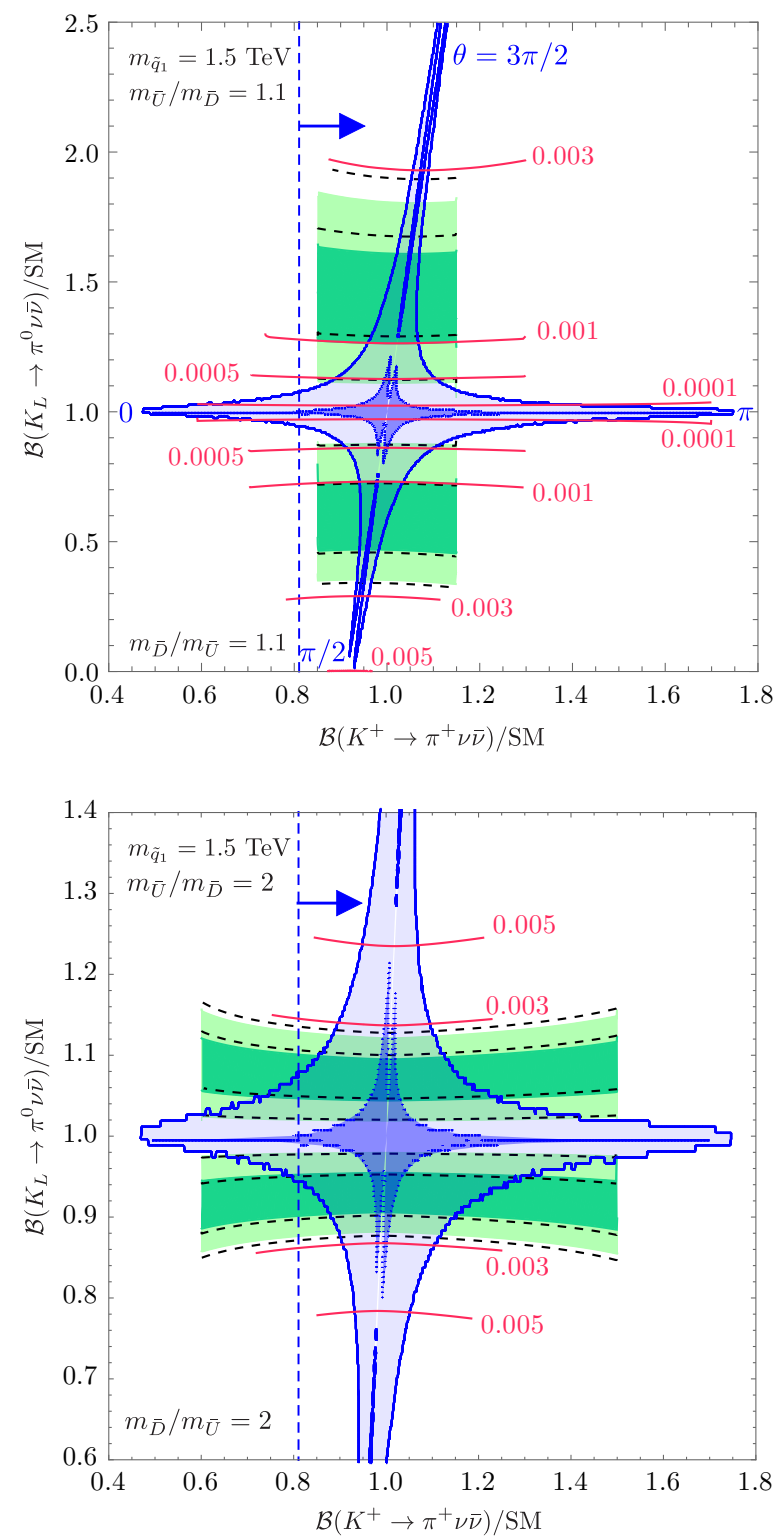

Figure 5. The correlation is shown in the Trojan penguin scenario. The light (dark) blue region requires a milder parameter tuning than $1 \%(10 \%)$ of the gluino mass and the $C P$ violating phase in order to suppress contributions to $\varepsilon_{K}$. The red contour represents the SUSY contributions to $\varepsilon_{K}^{\prime} / \varepsilon_{K}$, and the $\varepsilon_{K}^{\prime} / \varepsilon_{K}$ discrepancy is resolved at $1 \sigma(2 \sigma)$ within the dark (light) green region. The lightest squark mass is fixed to $1.5 \mathrm{TeV}$. In the top panel, $m_{\bar{D}} / m_{\bar{U}}=1.1\left(m_{\bar{U}} / m_{\bar{D}}=1.1\right)$ is used for $0<\theta<\pi$ $(\pi<\theta<2 \pi)$ to obtain a positive SUSY contribution to $\varepsilon_{K}^{\prime} / \varepsilon_{K}$. While, $m_{\bar{D}} / m_{\bar{U}}=2\left(m_{\bar{U}} / m_{\bar{D}}=2\right)$ is used for $0<\theta<\pi$ $(\pi<\theta<2 \pi)$ in the bottom panel. The region on the right side of the blue dashed lines are allowed by the current experimental measurements.

in the SM there is a large numerical cancelation between QCD-penguin and the $Z$-penguin contributions to $\varepsilon_{K}^{\prime} / \varepsilon_{K}$, a modified $Z$ flavour-changing $(s-d)$ interaction from NP can explain the current $\varepsilon_{K}^{\prime} / \varepsilon_{K}$ easily [32]. Then, the decay, $s \rightarrow d v \bar{v}$, proceeding through an intermediate $Z$ boson, is modified by NP. Therefore, the branching ratios of $K \rightarrow \pi v \bar{v}$ are likely to deviate from the SM predictions 
once the $\varepsilon_{K}^{\prime} / \varepsilon_{K}$ discrepancy between Eq. (1) and Eq. (3) is explained by the modified $Z$-coupling. They could be a signal to test the scenario. In the MSSM, such a scenario is also realized when the off-diagonal components of the trilinear SUSY-breaking couplings are large [33-35].

Such a signal is constrained by $\varepsilon_{K}$. The modified $Z$ couplings affect $\varepsilon_{K}$ via the so-called double penguin diagrams; the $Z$ boson mediates the transition with two flavour-changing $Z$ couplings. Such a contribution is enhanced when there are both left-handed and righthanded couplings because of the chiral enhancement of the hadronic matrix elements. An important point is that since the left-handed coupling is already present in the SM, the right-handed coupling must be constrained even without NP contributions to the left-handed one. Such interference contributions between the NP and the SM have been overlooked in the literature. References [35-37] have revisited the modified $Z$-coupling scenario including the interference contributions, and found the parameter regions allowed by the indirect $C P$ violation change significantly.

We find that similar to the previous section, the deviations of $\mathcal{B}(K \rightarrow \pi v \bar{v})$ from the SM values are determined by the necessary amount of the tuning in NP contributions to $\varepsilon_{K}$. We parametrize it by $\xi$ : A degree of the NP parameter tuning is represented by $1 / \xi$, e.g., $\xi=10$ means that the model parameters are tuned at the $10 \%$ level. The definition of $\xi$ is given in Ref. [36].

In Fig. 6, contours of the tuning parameter $\xi$ are shown for the simplified scenarios: LHS (all NP effects appear as left-handed), RHS (all NP effects appear as right-handed), ImZS (NP effects are purely imaginary), and LRS (leftright symmetric scenario) on the plane of the branching ratios of $K \rightarrow \pi v \bar{v}$ which are normalized by their SM predictions. We scanned the whole parameter space of the modified $Z$-coupling in each scenario, and selected the parameters where $\varepsilon_{K}^{\prime} / \varepsilon_{K}$ is explained at the $1 \sigma$ level. The experimental bounds from $\varepsilon_{K}, \Delta M_{K}$, and $\mathcal{B}\left(K_{L} \rightarrow \mu^{+} \mu^{-}\right)$are satisfied. In most of the allowed parameter regions, $\xi=O(1)$ is obtained. Thus, one does not require tight tunings in these simplified scenarios. In the figures, $\mathcal{B}\left(K_{L} \rightarrow \pi^{0} v \bar{v}\right)$ is smaller than the $\mathrm{SM}$ value by more than $30 \%$. On the other hand, $\mathcal{B}\left(K^{+} \rightarrow \pi^{+} v \bar{v}\right)$ depends on the scenarios. In LHS, we obtain $0<\mathcal{B}\left(K^{+} \rightarrow \pi^{+} v \bar{v}\right) / \mathcal{B}\left(K^{+} \rightarrow\right.$ $\left.\pi^{+} v \bar{v}\right)_{\mathrm{SM}}<1.8$. In RHS, $\mathcal{B}\left(K^{+} \rightarrow \pi^{+} v \bar{v}\right)$ is comparable to or larger than the SM value, but cannot be twice as large. In ImZS, the branching ratios are perfectly correlated and $\mathcal{B}\left(K^{+} \rightarrow \pi^{+} v \bar{v}\right)$ does not deviate from the SM one. In LRS, $\mathcal{B}\left(K_{L} \rightarrow \pi^{0} v \bar{v}\right)$ does not exceed about a half of the SM value. The more general situation is discussed in Ref. [36].

\section{Discussion and conclusions}

In this contribution, we introduced the current situation for the $\varepsilon_{K}^{\prime} / \varepsilon_{K}$ within the SM. The first lattice result and the improved perturbative calculations have shown the discrepancy between the predicted value and the data. Several NP models can explain the discrepancy of $\varepsilon_{K}^{\prime} / \varepsilon_{K}$, and then $\mathcal{B}(K \rightarrow \pi v \bar{v})$ are predicted to deviate from the SM predictions. We have presented the correlations between $\varepsilon_{K}^{\prime} / \varepsilon_{K}$,
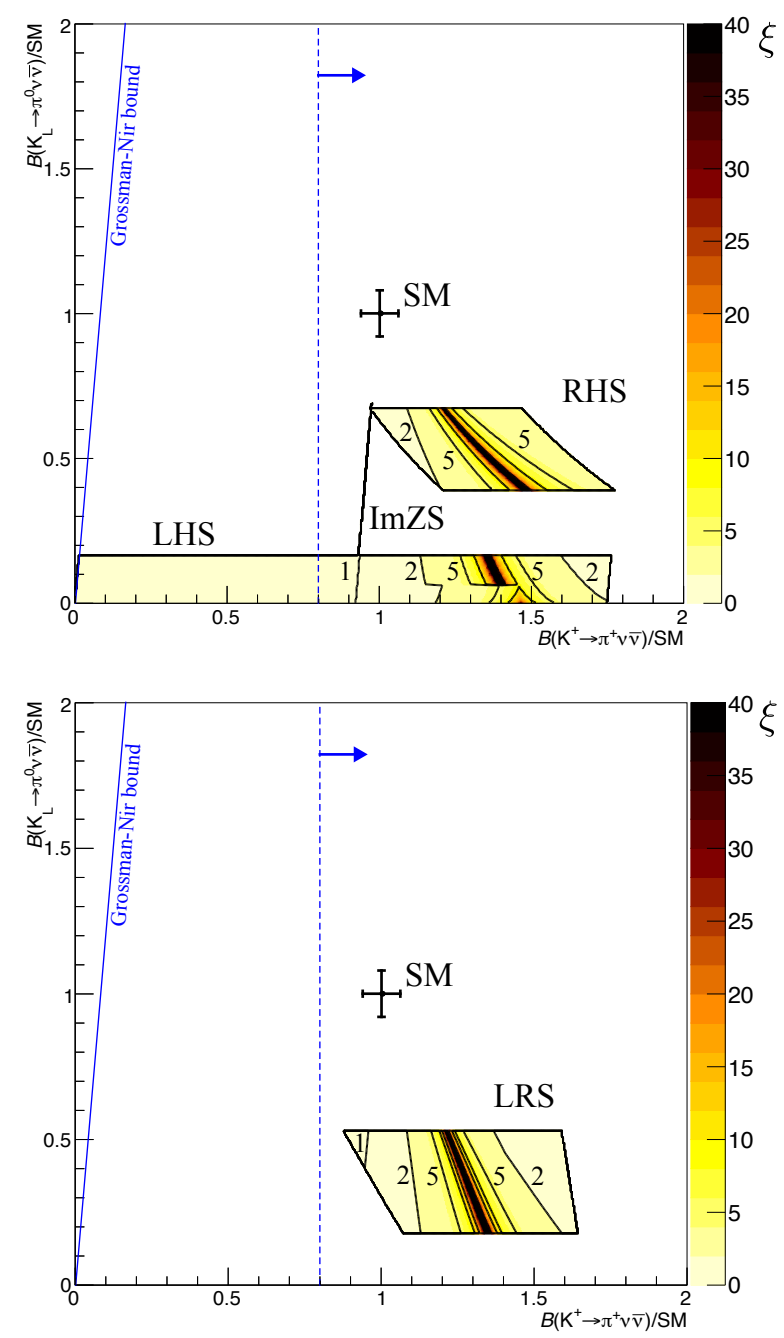

Figure 6. Contours of the tuning parameter $\xi$ are shown in the simplified modified Z-coupling scenarios: LHS, RHS, and ImZS (top panel) and LRS (bottom). In the colored regions, $\varepsilon_{K}^{\prime} / \varepsilon_{K}$ is explained at $1 \sigma$, and the experimental bounds of $\varepsilon_{K}, \Delta M_{K}$, and $\mathcal{B}\left(K_{L} \rightarrow \mu^{+} \mu^{-}\right)$are satisfied. The right region of the blue dashed line is allowed by the measurement of $\mathcal{B}\left(K^{+} \rightarrow \pi^{+} v \bar{v}\right)$ at $1 \sigma$. The NP scale is set to be $\mu=1 \mathrm{TeV}$.

$\mathcal{B}\left(K_{L} \rightarrow \pi^{0} v \bar{v}\right)$, and $\mathcal{B}\left(K^{+} \rightarrow \pi^{+} v \bar{v}\right)$ in the box dominated scenario and the $Z$-penguin dominated one. It is shown that the constraint from $\varepsilon_{K}$ produces different correlations between two NP scenarios. In the future, measurements of $\mathcal{B}(K \rightarrow \pi v \bar{v})$ will be significantly improved. The NA62 experiment at CERN measuring $\mathcal{B}\left(K^{+} \rightarrow \pi^{+} v \bar{v}\right)$ is aiming to reach a precision of $10 \%$ compared to the SM value already in 2018 [38]. In order to achieve 5\% accuracy more time is needed. Concerning $K_{L} \rightarrow \pi^{0} v \bar{v}$, the KOTO experiment at J-PARC aims in a first step at measuring $\mathcal{B}\left(K_{L} \rightarrow \pi^{0} v \bar{v}\right)$ around the SM sensitivity. Furthermore, the KOTO-step2 experiment will aim at 100 events for the SM branching ratio, implying a precision of $10 \%$ of this measurement [39]. Therefore, we conclude that when the $\varepsilon_{K}^{\prime} / \varepsilon_{K}$ discrepancy is explained by the NP contribution, NA62 experiment could probe whether a modified $Z$ coupling scenario is realized or not, and KOTO-step2 ex- 
periment can distinguish the box dominated scenario and the simplified modified $Z$-coupling scenario.

We should also comment on $K_{S} \rightarrow \mu^{+} \mu^{-}$, which proceeds via long-distance $C P$-conserving P-wave and shortdistance $C P$-violating $\mathrm{S}$-wave processes. Since the decay rate is dominated by the former, whose uncertainty is large, the sensitivity to the short-distance contributions is diminished. However, it is pointed out that the short-distance contribution is enhanced through an interference between the $K_{L}$ and $K_{S}$ states in the neutral kaon beam [40]. Therefore, one can also distinguish the NP scenarios using the correlation with $K_{S} \rightarrow \mu^{+} \mu^{-}$. Such a correlation has been investigated in the box dominated scenario (with large $\tan \beta$ ) [41] and the modified $Z$-coupling scenario $[35,40]$.

\section{Acknowledgment}

I would like to thank Veronika Chobanova, Andreas Crivellin, Giancarlo D’Ambrosio, Motoi Endo, Toru Goto, Miriam Lucio Martinez, Diego Martinez Santos, Satoshi Mishima, Ulrich Nierste, Isabel Suarez Fernandez, Paul Tremper, Daiki Ueda, and Kei Yamamoto for fruitful collaborations on the presented work. I also want to warmly thank the organizers of FCCP 2017 for inviting and giving me the opportunity to present these results in a great conference.

\section{References}

[1] A. J. Buras and J. M. Gérard, Nucl. Phys. B 264, 371 (1986); A. J. Buras, J. M. Gérard and W. A. Bardeen, Eur. Phys. J. C 74, 2871 (2014) [arXiv:1401.1385 [hep$\mathrm{ph}]$.

[2] T. Blum et al., Phys. Rev. Lett. 108, 141601 (2012) [arXiv:1111.1699 [hep-lat]]; T. Blum et al., Phys. Rev. D 86, 074513 (2012) [arXiv:1206.5142 [hep-lat]]; T. Blum et al., Phys. Rev. D 91, no. 7, 074502 (2015) [arXiv:1502.00263 [hep-lat]];

[3] Z. Bai et al. [RBC and UKQCD Collaborations], Phys. Rev. Lett. 115, no. 21, 212001 (2015) [arXiv:1505.07863 [hep-lat]].

[4] S. Bertolini, J. O. Eeg, M. Fabbrichesi and E. I. Lashin, Nucl. Phys. B 514, 93 (1998) [hep$\mathrm{ph} / 9706260]$.

[5] E. Pallante and A. Pich, Nucl. Phys. B 592, 294 (2001) [hep-ph/0007208]; E. Pallante, A. Pich and I. Scimemi, Nucl. Phys. B 617, 441 (2001) [hep-ph/0105011].

[6] T. Hambye, S. Peris and E. de Rafael, JHEP 0305, 027 (2003) [hep-ph/0305104].

[7] A. J. Buras and J. M. Gérard, JHEP 1512, 008 (2015) [arXiv:1507.06326 [hep-ph]].

[8] A. J. Buras and J. M. Gerard, Eur. Phys. J. C 77, no. 1, 10 (2017) [arXiv:1603.05686 [hep-ph]].

[9] A. J. Buras, M. Gorbahn, S. Jäger and M. Jamin, JHEP 1511, 202 (2015) [arXiv:1507.06345 [hep-ph]].

[10] T. Kitahara, U. Nierste and P. Tremper, JHEP 1612, 078 (2016) [arXiv:1607.06727 [hep-ph]].
[11] L. K. Gibbons et al., Phys. Rev. Lett. 70, 1203 (1993).

[12] G. D. Barr et al. [NA31 Collaboration], Phys. Lett. B 317, 233 (1993).

[13] V. Fanti et al. [NA48 Collaboration], Phys. Lett. B 465, 335 (1999) [hep-ex/9909022]; J. R. Batley et al. [NA48 Collaboration], Phys. Lett. B 544, 97 (2002) [hep-ex/0208009].

[14] A. Alavi-Harati et al. [KTeV Collaboration], Phys. Rev. Lett. 83, 22 (1999) [hep-ex/9905060]; E. Abouzaid et al. [KTeV Collaboration], Phys. Rev. D 83, 092001 (2011) [arXiv:1011.0127 [hep-ex]].

[15] C. Patrignani et al. [Particle Data Group], Chin. Phys. C 40, no. 10, 100001 (2016).

[16] H. Gisbert and A. Pich, arXiv:1712.06147 [hep-ph].

[17] V. Cirigliano, A. Pich, G. Ecker and H. Neufeld, Phys. Rev. Lett. 91, 162001 (2003) [hep-ph/0307030].

[18] L. Lellouch and M. Luscher, Commun. Math. Phys. 219, 31 (2001) [hep-lat/0003023].

[19] G. Colangelo, J. Gasser and H. Leutwyler, Nucl. Phys. B 603, 125 (2001) [hep-ph/0103088].

[20] R. Garcia-Martin, R. Kaminski, J. R. Pelaez, J. Ruiz de Elvira and F. J. Yndurain, Phys. Rev. D 83, 074004 (2011) [arXiv:1102.2183 [hep-ph]].

[21] X. Feng, arXiv:1711.05648 [hep-lat].

[22] C. Lehner, E. Lunghi and A. Soni, Phys. Lett. B 759, 82 (2016) [arXiv:1508.01801 [hep-ph]].

[23] T. Kitahara, U. Nierste and P. Tremper, Phys. Rev. Lett. 117, no. 9, 091802 (2016) [arXiv:1604.07400 [hep-ph]].

[24] A. L. Kagan and M. Neubert, Phys. Rev. Lett. 83, 4929 (1999) [hep-ph/9908404]; Y. Grossman, M. Neubert and A. L. Kagan, JHEP 9910, 029 (1999) [hepph/9909297].

[25] A. Crivellin and M. Davidkov, Phys. Rev. D 81, 095004 (2010) [arXiv:1002.2653 [hep-ph]].

[26] A. J. Buras, M. Jamin, M. E. Lautenbacher and P. H. Weisz, Nucl. Phys. B 400, 37 (1993) [hepph/9211304]; A. J. Buras, M. Jamin and M. E. Lautenbacher, Nucl. Phys. B 400, 75 (1993) [hepph/9211321]; M. Ciuchini, E. Franco, G. Martinelli and L. Reina, Nucl. Phys. B 415, 403 (1994) [hep$\mathrm{ph} / 9304257]$.

[27] J. A. Bailey et al. [SWME Collaboration], Phys. Rev. D 92, no. 3, 034510 (2015) [arXiv:1503.05388 [heplat]].

[28] G. Aad et al. [ATLAS Collaboration], JHEP 1510 (2015) 054 [arXiv:1507.05525 [hep-ex]].

[29] The ATLAS collaboration [ATLAS Collaboration], ATLAS-CONF-2016-078.

[30] CMS Collaboration [CMS Collaboration], CMSPAS-SUS-16-015.

[31] A. Crivellin, G. D'Ambrosio, T. Kitahara and U. Nierste, Phys. Rev. D 96, no. 1, 015023 (2017) [arXiv:1703.05786 [hep-ph]].

[32] A. J. Buras, F. De Fazio and J. Girrbach, Eur. Phys. J. C 74, no. 7, 2950 (2014) [arXiv:1404.3824 [hep-ph]]; 
A. J. Buras, D. Buttazzo and R. Knegjens, JHEP 1511, 166 (2015) [arXiv:1507.08672 [hep-ph]]; A. J. Buras, JHEP 1604, 071 (2016) [arXiv:1601.00005 [hep-ph]].

[33] M. Tanimoto and K. Yamamoto, PTEP 2016, no. 12, 123B02 (2016) [arXiv:1603.07960 [hep-ph]].

[34] M. Endo, S. Mishima, D. Ueda and K. Yamamoto, Phys. Lett. B 762, 493 (2016) [arXiv:1608.01444 [hepph]].

[35] M. Endo, T. Goto, T. Kitahara, S. Mishima, D. Ueda and K. Yamamoto, arXiv:1712.04959 [hep-ph].

[36] M. Endo, T. Kitahara, S. Mishima and K. Yamamoto, Phys. Lett. B 771, 37 (2017) [arXiv:1612.08839 [hep$\mathrm{ph}]$.

[37] C. Bobeth, A. J. Buras, A. Celis and M. Jung, JHEP 1707, 124 (2017) [arXiv:1703.04753 [hep-ph]].
[38] E. Cortina Gil et al. [NA62 Collaboration], JINST 12, no. 05, P05025 (2017) [arXiv:1703.08501 [physics.ins-det]].

[39] Talk by H. Nanjo in International workshop on physics at the extended hadron experimental facility of J-PARC, KEK Tokai, Japan, 5-6 March 2016; Talk by G. Ruggiero in EPS Conference on High Energy Physics, Venice, Italy, 5-12 July 2017.

[40] G. D'Ambrosio and T. Kitahara, Phys. Rev. Lett. 119, no. 20, 201802 (2017) [arXiv:1707.06999 [hep$\mathrm{ph}]$.

[41] V. Chobanova, G. D’Ambrosio, T. Kitahara, M. Lucio Martinez, D. Martinez Santos, I. S. Fernandez and K. Yamamoto, arXiv:1711.11030 [hep-ph]. 